\title{
Case of congenital lobar lung emphysema
}

\author{
Bulegenova Minira* Makhneva Anna and Bekisheva Aigul
}

*Correspondence: mbulegenova@yandex.ru

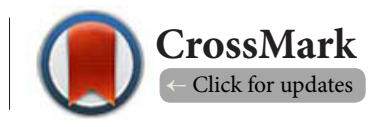

Department of Pathology, Pediatric and Pediatric Surgery Research Center, Kazakhstan.

\begin{abstract}
The authors describe a case of congenital lobar emphysema in child at the age of 45 days and discuss the clinical manifestations as well as the features of histological diagnostic of the disease. This case is of particular interest because of the rarity of the pathology as well as the fact that congenital lobar emphysema developed as a result of combination of two anomalies of the bronchi: mucosal folding and muscular layer hypoplasia.
\end{abstract}

Keywords: Congenital lobar emphysema, clinical manifestation, muscular layer hypoplasia, lung malformation

\section{Introduction}

Congenital lobar emphysema (CLE) is a malformation characterized by excessive air content in the lung lobe, found mostly in the early childhood. This pathology is reported quite rare and its true incidence has not been established.

CLE is more common in boys. Concerning localization it affects predominantly the upper (42.2\%) and the medium lobe (35.3\%) of the left lung, less frequently the upper lobe of the right lung $(20.7 \%)$ and in very rare cases the lower lobes (0.9\%) [1].

The formation of CLE may be caused by abnormal lobar bronchus development, most often it is a cartilage defect, less frequently a muscular coat defect, mucous membrane fold, stenosis, compression of the abnormally located vessel, tumor etc [2]. However, in $50-55 \%$ of cases it is not possible to identify the etiology of the disease [3-5].

The severity of clinical manifestations of the CLE depends on the hyperventilation degree in the affected lobe. The most severe forms are manifested during the first days of the child's life as dyspnea, cyanosis, apnea episodes, which can be fatal within a short time. Less severe forms of the disease are diagnosed later - at the age of 2-3 months. The pathology is manifested by cough, shortness of breath. Sick children have a tendency to the inflammatory diseases of the bronchopulmonary system, they often lag behind in physical development [6].

Depending on the manifestation grade of the lobar emphysema and the severity of the clinical manifestations there can be the following forms: decompensated, subcompensated and compensated form of the disease [3].

\section{Clinical observation}

The child S., 45 days was admitted to the Pediatrics and Pediatric
Surgery Research Center with a diagnosis of congenital lung malformation. Congenital emphysema of the left lung upper lobe P28.8. Postnatal bilateral pneumonia of moderate severity, acute course. Respiratory distress of 1-2 grade.

From the life anamnesis it is known, that the child was born after $2^{\text {nd }}$ pregnancy, $2^{\text {nd }}$ operative delivery. In mother: aggravated obstetric anamnesis, convergent strabismus, insolvent scar on the uterus. Ventriculomegaly in fetus. Birth weight 3564 $\mathrm{g}$ height $-53 \mathrm{~cm}$. Apgar score of 6-8 points. On the fourth day of life the respiratory disorders appeared, diagnosed: Cerebral ischemia. Stridor.

On the tenth day of life a deterioration of the general condition was detected, the respiratory failure appeared. During twelve days the child was given a symptomatic treatment with a diagnosis of acute community-acquired pneumonia with bilateral obstructive syndrome. The condition deteriorated progressively due to the rise of respiratory distress signs.

At the age of one month a survey radiography was performed, which revealed a mediastinal shift to the right.

CT scan data at the place of residence (city of Uralsk): bilateral pneumonia with abscess formation on the right side (?). Right lung hypoplasia.

A teleconference with the PPSRC was arranged and the patient's transfer was recommended. The signs of the respiratory distress were detected at the initial examination in the PPSRC: auxiliary muscles involvement in breathing, shortness of breath. Neurological status: prevalence of depression, nasolabial triangle cyanosis associated with anxiety. Auscultation: weakened breathing in all fields, no wheezing.

Computer tomography of the thorax: congenital malformation of the lung. Stenosis of the left upper lobar bronchus. Congenital emphysema of the left lung upper lobe. 
Minira et al. Journal of Histology \& Histopathology 2014,

http://www.hoajonline.com/journals/pdf/2055-091X-1-13.pdf

Operation: Left sided thoracotomy. Left upper lobe resection. Left sided thoracostomy.

\section{Materials and methods}

Surgical material was cut in pieces of size $1.0 \times 1.0 \times 0.5 \mathrm{~cm}$, fixed in $10 \%$ neutral buffered formalin, dehydrated in the solution based on isopropyl alcohol IsoPrep (BioVitrum, Russia), embedded in a paraffin medium HISTOMIX ${ }^{\circledR}$ EXTRA, $54^{\circ} \mathrm{C}$ (BioVitrum, Russia); the paraffin sections 2 microns in thickness were prepared on the microtome Microm HM340E (Thermo SCIENTIFIC, Germany), clarified in the solution «Bio-Clear» (Bio-Optica, Italy), stained with Mayer's hematoxylin (BioOptica, Italy) and Eosin Y 1\% aqueous solution (Bio-Optica, Italy) using the common method.

\section{Results}

Within the pathomorphological study the following changes were revealed: visceral pleura with local fiber separations, numerous empty spaces were found in subpleural area -places of visceral pleura detachment from the lung tissue. Most of alveoli had lumens of different sizes, among which were dramatically enlarged ones. Interalveolar septum were thinned and stretched, ruptured in some places with a formation of microcysts in these areas. In the lumen of some alveoli the eosinophilic masses with the admixture of macrophages and erythrocytes were detected. Small foci of atelectasis containing alveoli with slotted openings were seen as well. Interalveolar septum in these areas were broad. The cavities lined on the one side with prismatic epithelium, on the other side with alveolar were seen frequently. The epithelial lining of the bronchial tubes formed folds, in some places the muscle plate under the mucous base was missing. The vessels had irregular blood supply, red blood cells in their lumen were lysed, the focal hemorrhages could be seen. The layered structures in the form of perivascular sclerosis were identified (see Figures 1 and 2).

Treatment in the postoperative period: artificial lung ventilation, antibiotics, infusion therapy.

Satisfactory condition in the postoperative period, the symptoms of respiratory distress cut short. Weight $5200 \mathrm{~g}$, height $59 \mathrm{~cm}$. Discharged with recommendations.

\section{Discussion and conclusion}

The case described in this article is of particular interest for pathologists and pediatricians due to the fact that congenital lobar emphysema in patient was the result of combination of the two bronchial anomalies: mucosal folding and muscular layer hypoplasia that from the first days of life caused a respiratory distress, which determined the severity of the disease. It should be noted that early surgery in case of congenital lobar emphysema helps to avoid irreversible consequences. In the presented case a possible cause of delayed diagnosis of this disease was gradual emphysema development in the child.
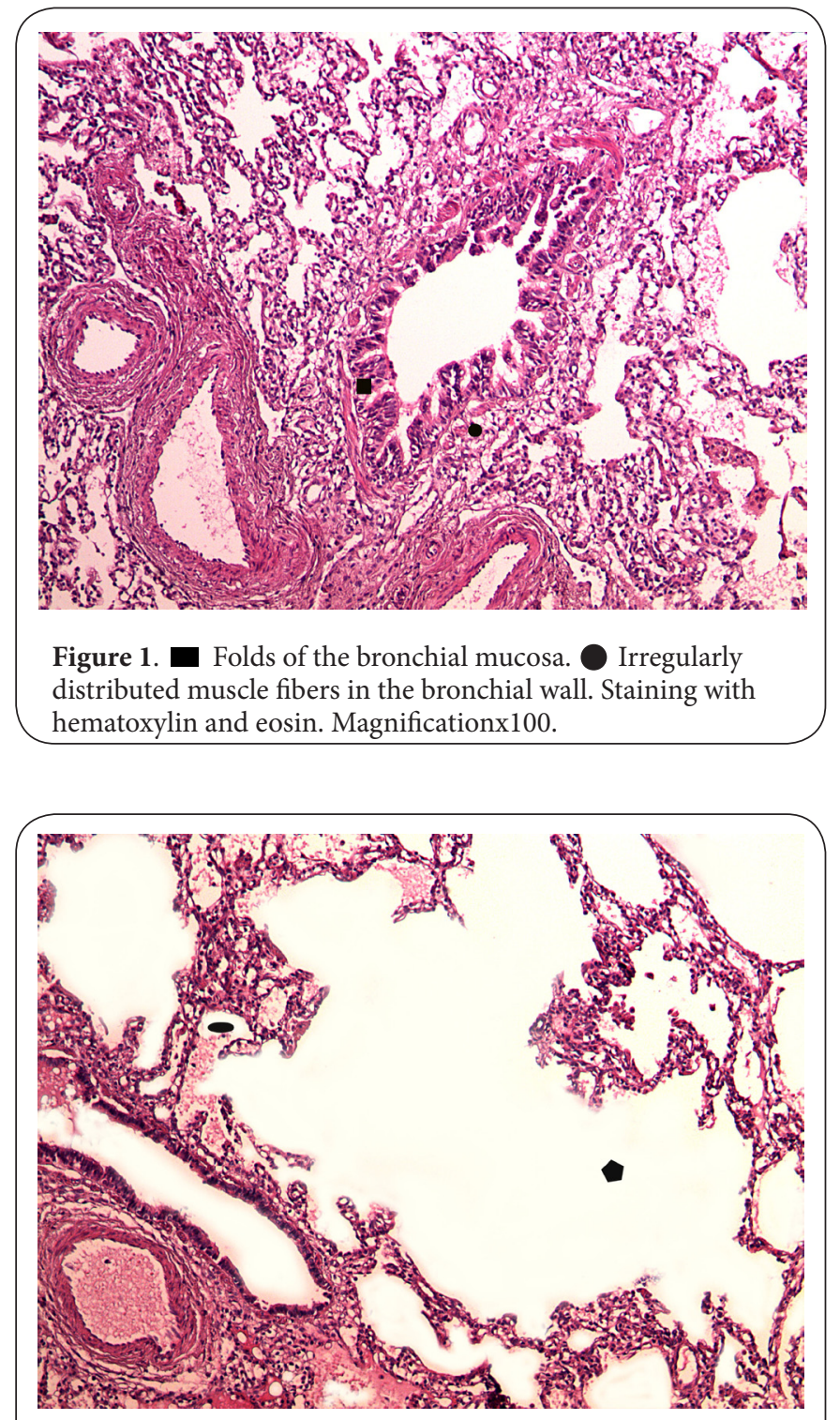

Figure 2. Emphysematously enlarged alveoli, interalveolar septum ruptured with microcyst formation. $\rightarrow$ The red blood cells isolated in the lumen of some alveoli. Staining with hematoxylin and eosin. Magnificationx100.

Competing interests

The authors declare that they have no competing interests.

Authors' contributions

\begin{tabular}{|l|c|c|c|}
\hline Authors' contributions & BM & MA & BA \\
\hline Research concept and design & $\checkmark$ & $\checkmark$ & $\checkmark$ \\
\hline Collection and/or assembly of data & -- & -- & $\checkmark$ \\
\hline Data analysis and interpretation & -- & $\checkmark$ & -- \\
\hline Writing the article & $\checkmark$ & $\checkmark$ & $\checkmark$ \\
\hline Critical revision of the article & $\checkmark$ & -- & -- \\
\hline Final approval of article & $\checkmark$ & -- & -- \\
\hline Statistical analysis & -- & -- & -- \\
\hline
\end{tabular}


Minira et al. Journal of Histology \& Histopathology 2014,

\section{Publication history}

EIC: Giuseppe Musumeci, University of Catania, Italy.

Received: 05-Sep-2014 Final Revised: 01-Oct-2014

Accepted: 18-Nov-2014 Published: 25-Nov-2014

\section{References}

1. Stocker JT, Madewell JE and Drake RM. Congenital cystic adenomatoid malformation of the lung. Classification and morphologic spectrum. Hum Pathol. 1977; 8:155-71. | Article I PubMed

2. Landing $B H$ and Dixon LG. Congenital malformations and genetic disorders of the respiratory tract (larynx, trachea, bronchi, and lungs). Am Rev Respir Dis. 1979; 120:151-85. | PubMed

3. Isakov LF, Stepanov EA and Michelson BA. Surgical diseases in children. Medicine, Moscow. 2008; 289-290.

4. Bhandary A. Congenital malformations of the lung and the airway Pediatric pulmonology. Mosby, Inc. 2005; 60-76.

5. Berrocal T, Madrid C, Novo S, Gutierrez J, Arjonilla A and Gomez-Leon $\mathrm{N}$. Congenital anomalies of the tracheobronchial tree, lung, and mediastinum: embryology, radiology, and pathology. Radiographics. 2004; 24:17. | Article | PubMed

6. Rozinova LV, Sorokin EB and Kostjuchenko MB. Williams Syndrome-lobar emphysema and Campbell - rare lung malformations with a single pathogenetic basis. Difficult patient. 2009; 9:48.

\section{Citation:}

Minira B, Anna M and Aigul B. Case of congenital lobar lung emphysema. J Histol Histopathol. 2014; 1:13. http://dx.doi.org/10.7243/2055-091X-1-13 